\title{
LA DOCENCIA COMO PRÁCTICA POLÍTICA EN COLOMBIA: MÁS QUE FORMACIÓN, CARRERA Y REMUNERACIÓN, MÁS QUE ENSEÑAR, EDUCAR Y APRENDER
}

\author{
A DOCÊNCIA COMO PRÁTICA POLÍTICA NA COLÔMBIA: MAIS QUE FORMAÇÃO, \\ CARREIRA E REMUNERAÇÃO, MAIS QUE ENSINAR, EDUCAR E APRENDER
}

\section{TEACHING AS POLITICAL PRACTICE IN COLOMBIA: MORE THAN TRAINING, CAREER AND REMUNERATION, MORE THAN TEACHING, EDUCATING AND LEARNING}

Luis Fernando Vásquez Zora*

Todo sistema de educación es una forma política de mantener o de modificar la adecuación de los discursos, con los saberes y los poderes que implican. (FOUCAULT, 1992, p. 38)

\begin{abstract}
Resumen: Más que referir la docencia como aquella ocupación, profesión o profesionalidad a través de la cual un sujeto social es formado para enseñar, educar y/o posibilitar aprendizajes, este artículo describe como esta actividad social es resultado de un especializado conjunto de experiencias políticas discontinuas -disciplinarias, normalizadoras y de control-, por las cuales nuestra sociedad en tiempos y espacios específicos, coloca en juego determinados órdenes de saber, específicas modalidades de poder con la pretensión de imponer prácticas docentes singulares como elaboración de sofisticadas formas de gobierno social. El instrumental teórico-metodológico corresponde al concepto de políticas docentes, describe las condiciones de formación, existencia y desaparición de la docencia como práctica producida bajo la intervención de positividades específicas que definen y formulan determinados mecanismos y procedimientos de poder por los cuales se intenta tornar la docencia en práctica social de enseñanza, educación y aprendizaje. El concepto de políticas docentes es un instrumental que busca exhibir la docencia como conjunto de las prácticas religiosas y morales, normalizadoras y educativas, productivas y técnico-científicas, profesionalizantes y de control, que realizadas entre instancias y sujetos, dan lugar a las singulares funciones sociales de reunir, de instruir, de disciplinar, de educar, de capacitar, de estimular innovaciones y competencias como conjunto de efectos específicos de gobierno social. El artículo demuestra que la docencia se corresponde a la elaboración social de múltiples y sofisticadas prácticas políticas, formadas por estrategias y tácticas de saber y de poder, a través de las cuales se calcula, prescribe, organiza, realizan y relacionan sujetos e instituciones bajo la pretensión de conformar docencias como objeto e instrumento de disciplina, de mediación, de eficacia técnicocientífica, de emprendedurismo, de gestión y control de competencias como modalidades de gobierno social. Finalmente, resalta que en los docentes se halla el poder de apropiarse, atribuirse y proponer una docencia trasgresora de sí y de los otros.
\end{abstract}

Palabras clave: Políticas docentes. Saber. Poder. Prácticas.

* Universidade Federal de Minas Gerais - UFMG. E-mail: luisfernandovasquez@yahoo.com 
Resumo: Mais do que se referir à docência como ocupação ou profissão na qual um sujeito social é formado para ensinar, educar ou para possibilitar aprendizagens, este artigo descreve como esta atividade social é resultado de um conjunto especializado de experiências históricas e políticas descontínuas - disciplinadoras, normalizadoras e de controle - pelas quais nossa sociedade, em tempos e espaços específicos, coloca em jogo determinadas ordens de saber e modalidades de poder que pretendem impor práticas docentes singulares e desenvolver formas sofisticadas de governo social.

O instrumento teórico-metodológico corresponde ao conceito de políticas docentes, o qual descreve as condições de formação, existência y desaparecimento da docência a partir da intervenção de positividades específicas que definem e formulam certos mecanismos e procedimentos de poder pelos quais se busca transformar a docência uma prática social de ensino, educação e aprendizagem.

O conceito de políticas docentes é um instrumento que busca mostrar a docência como um conjunto de práticas religiosas e morais, normalizadoras e educativas, produtivas e técnico-científicas, profissionalizantes e de controle que, realizadas entre instâncias e sujeitos, dão lugar às funções sociais próprias de reunir, de instruir, de disciplinar, de educar, de capacitar, de estimular inovações e competências como conjunto de efeitos do governo social. O artigo demostra que a docência corresponde a múltiplas práticas políticas, formadas por estratégias e táticas de saber e poder através das quais se calculam, prescrevem, organizam, realizam e relacionam sujeitos e instituições a partir da intenção de configurar a docência como objeto e instrumento de disciplina, de mediação, de eficácia técnicocientífica, empresarial, de gestão e de competências como tecnologias especializadas de governo social. Finalmente, ressalta que nos docentes se encontra o poder de apropriar-se, atribuir-se e devir uma docência transgressora, que se reinvente para si mesma e para os outros.

Palavras-chave: Políticas docentes. Saber. Poder. Práticas.

Abstract: Rather than refer to teaching as that occupation, profession or professionalism through which a social subject is trained to teach, educate and / or facilitate learning, this article describes how this social activity is the result of a specialized set of discontinued historical experiences and policies modalities -disciplinarian, normalizing, and of control-, which our society in specific times and places, set in motion certain orders of knowledge, specific forms of power with the aim of imposing unique teaching practices as development of sophisticated forms of social government. The theoreticalmethodological instrument corresponds to the concept of teachers' policies, describes the conditions of formation, existence and disappearance of teaching as a practice produced under the action of specific positive reactions that define and formulate certain mechanisms and procedures of power which strive to do a teaching in social practice teaching, education and learning. The concept of teachers' policies is an instrument that seeks to showcase teaching as a set of religious and moral practices, normalizing and educational, productive and technical-scientific, professionalizing and of control, which performed between instances and subjects, result in the unique social functions to gather, to instruct, to discipline, to educate, to train, to stimulate innovation and skills as a set of specific effects of social government. The article shows that teaching pertains to multiple practices that are historical, political and social, consisting of strategies and tactics of knowledge and power, through which institutions are calculated, prescribed, organized, conducted and related under the pretense of conforming teaching practices to be subject and instruments to disciplinary, mediating techniques, technical-scientific, business efficiency, management and competence as specialized technologies of the government. Finally, it emphasizes that teachers have the power to appropriate, attribute and become a transgressive teaching of oneself and of others.

Keywords: Teachers’ policies. Knowledge. Power. Practices. 


\section{Introducción}

¿Qué tipo de experiencia política es la docencia ${ }^{1}$ para que nuestra sociedad en espacios y tiempos disímiles reúna en esta actividad determinados órdenes de saber y especializadas modalidades de poder con la pretensión de establecer prácticas sociales que definan singulares formas de gobierno de sí y de los otros? ¿Cómo se han conformado en determinados periodos históricos un conjunto específico de modalidades de enseñanza disciplinaria -religiosas, morales e instruccionales-; normalizadoras educativas y científico-técnicas-; de controlprofesionales, de gestión y de competencias-, para esta actividad social? ¿Significan éstos diferentes órdenes de saber y de poder la elaboración histórica y social de sofisticadas prácticas de gobierno de si y de los otros, a través de especializadas modalidades políticas de aquel que sabe, piensa, dice y ordena? $¿$ Es la docencia en nuestras sociedades una elaborada práctica política?

Si es del lado de los saberes, la actividad docente es objeto de toda

\footnotetext{
1 "La palabra docencia, proviene del latín docere (enseñar) la cual nos dio las palabras: doctrina, doctor, documento y dócil. Docere tiene la raíz indoeuropea: dek- que quiere decir pensamiento o aceptación. Otras palabras derivadas de la raíz indoeuropea dekson: El verbo griego ठокєıv (dokein = opinar) que nos dio las palabras: dogma, ortodoxo y paradoja. // El verbo latino decet (en tercera persona ya que es impersonal), 'ser apropiado’ y de ahí: decente. // La palabra latina dignus: digno y derivados dignidad, dignatario, dignificación, indignar, etc. // El verbo latino disco: discípulo y disciplina. // La palabra griega ঠокós (dokos = viga) y de ahí diplodoco (dos vigas), en relación a las vértebras de este animal prehistórico.

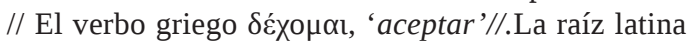
deuk (guiar) de la cual encontramos en palabras como acueducto, duque, conducir, educar, ducha, dúctil, y compuestos del verbo latino duco como deducir, seducir, traducir, inducir, abducir, aducir o introducir". Miguel, Raimundo de. (2001). Nuevo diccionario latino español etimológico. Madrid: Visor.
}

una grilla de conocimientos filosóficos (SÓCRATES, 1985; PLATÓN, 1988, 1999; ARISTÓTELES, 1999; QUINTILIANO, 1916, LOCKE, 2012; VIVES, 1923), teológicos (AGUSTÍN, 1953; TOMÁS, 2000; LOYOLA, 1977; CALASANZ, 2007; LA SALLE, 2001; BOSCO, 2003; entre otros autores), pedagógicos (COMENIO, 1998; PESTALOZZI, 1889; BOVET, 2007; CLAPARÈDE, 2007; etc.), psicológicos (MONTESSORI, 2003; PIAGET, 2001, etc.), sociológicos (ROUSSEAU, 2000; KANT, 2000; DURKHEIM, 1979; DEWEY, 1977; DECROLY, 2006), técnico-científicos, administrativos y de gestión (FAURE, 1973; HAYEK, 1978; SCHULTZ, 1968; DELORS, 1996; UNESCO, 2015).

Si es del lado de las modalidades de poder esta actividad ha pasado, entre otras prácticas de gobierno, por modalidades disciplinarias, centradas en la elaboración de técnicas que pretenden imponer comportamientos y conductas a través del dominio del cuerpo y del espacio; de normalización, a través de modalidades de padronización y homogenización; de control, como especializada técnica de gobierno a distancia de sujetos y objetos a partir de la gestión de sí, como forma de dependencia de los otros. El control reclama como acción de dominio, el conocimiento de los otros para poder gobernarse así mismo.

Si es desde las prácticas, entre las figuras de la docencia conformadas por la integración de saberes específicos y de determinadas modalidades de poder, encontramos las prácticas del maestro-padre (FAVACHO, 2012; MARTÍNEZ, 1982; ZORA, 2015), las del preceptor e instructor (ROUSSEAU, 2000; LOCKE, 2012; MARTÍNEZ, 1982; ZORA, 2015), las del educador (ENGUITA, 1998, 2004; NÓVOA, 2009, 2014; TARDIF, 2012; ZORA, 2015), las del curriculista (MARTÍNEZ; NOGUERA; CASTRO, 
1989; ZORA, 2015), las del “ciber-antropo docente" o del capacitador tecno-científico (FAURE, 1973; ZORA, 2015), las del agente cultural (FECODE, 1982; TAMAYO, 2006; ZULUAGA, 1987), las de control, gestor de aprendizajes, innovaciones y competencias (ZORA, 2015). La docencia es, por lo tanto, la invención de una relación social y política, resultado de una serie de experiencias históricas discontinuas, orientadas por saberes y conducidas por poderes con la pretensión de imponer especializadas prácticas de gobierno de sí y de los otros.

A la relación entre saberes y poderes como productores de prácticas singulares de la docencia, las denominamos aquí: “políticas docentes” (ZORA, 2015, p. 22), y son el resultado de específicas modalidades de gobierno, comprendido como el juego de un conjunto determinado de saberes, de estrategias y tácticas que buscan imponer a través de ciertos fines y medios la elaboración de prácticas docentes determinadas.

Las políticas docentes se corresponden a específicos órdenes de saber y modalidades de poder, son prácticas que transcurren por experiencias disciplinarias a partir de la integración de elementos filosóficos, religiosos y morales; normalizadoras, desde modelos productivos y científico-técnicos; de control, en tanto, dispositivo que basa el gobierno social y educativo desde el profesionalismo, como desempeño de sí por la gestión, la calidad y recientemente, la docencia como práctica de excelencia (MEN, 2015).

Como herramienta teóricometodológica, las políticas docentes (ZORA, 2015), se proponen como instrumental de análisis y descripción de los distintos regímenes de formación, existencia y desaparecimiento de saberes y poderes que integran y definen la docencia como especializada modalidad de gobierno social.
En cuanto instrumento metodológico, plantea la analítica de las prácticas discursivas ${ }^{2}$ que integran las discontinuas figuras de la docencia, las modalidades a través de las cuales, entre instituciones y sujetos son establecidos mecanismos y procedimientos, estrategias y tácticas, fines y medios por los que son formadas singulares maneras de pensar, de ver y de decir como pretensión de imposición de modelos de gobierno de sí y de la sociedad a través de tecnologías específicas como la disciplina y la enseñanza, la normalización y la educación, el control y los aprendizajes.

Posteriormente, se mostrarán algunas modalidades de la docencia en Colombia, presentando unas cuantas prácticas políticas que corresponden a una investigación mayor (ZORA, 2015), y que para el presente artículo comprenden las figuras del maestro doctrinante, del preceptor, del maestro instructor moral, del maestro medio, del Ciber-antropo docente, del maestro agente cultural, del docente profesional de gestión y control.

En las consideraciones finales se exhibe la docencia como una sofisticada invención histórica, una tecnología de gobierno elaborada por especializados saberes y poderes. Entre algunas de las cuestiones que se discuten en el presente trabajo se hallan: ¿Cuáles tipos prácticas docentes son producidas por la integración de saberes filosóficos, teológicos, pedagógicos, sicológicos, médicos, biológicos, administrativos, científico-técnicos y de gestión? ¿Qué específicas relaciones de poder atraviesan la docencia como práctica social? ¿Qué tipos de sujetos políticos conforman las

\footnotetext{
${ }^{2}$ Por prácticas discursivas se comprende el ejercicio de “descubrir todo ese dominio de las instituciones, de los procesos económicos, de las relaciones sociales sobre las cuales puede articularse una formación discursiva”. (Foucault. 1979, p. 276).
} 
relaciones de saber y de poder que se integran en específicas prácticas docentes?

\section{Las políticas docentes como herramienta teórico-metodológica}

Quien enseña anuda, espera o desea a veces anudar entre sí mismo y quien o quienes lo escuchan un lazo, que es el saber común, de la herencia, de la tradición, lazo que puede ser también de reconocimiento personal o de amistad. Sea como fuere, entre su poder y su saber trasmite una enseñanza en la filiación a un orden de saber y de poder. (FOUCAULT, 2010, p. 40)

Más allá de denominar la docencia como ocupación, profesión o como actividad profesional que tiene por función la enseñanza, la educación o el aprendizaje sobre planes de producción de un sujeto social al que le es definido una formación inicial y continua, una carrera y una remuneración. A diferencia, las políticas docentes se proponen el análisis político de la docencia como práctica de gobierno, esto es, conforman el instrumental teórico-metodológico de análisis de la actividad como sofisticada tecnología social, integrada por saberes, que define alcances, límites, contenidos, así como procedimientos y mecanismos de poder (FOUCAULT, 2004, 2006), por los cuales la sociedad intenta conformar un conjunto singular de prácticas que posibiliten la implementación de un modelo específico de gobierno de la sociedad en una historicidad determinada.

De esta manera, las políticas docentes son la analítica de las condiciones de surgimiento, existencia y desaparecimiento del arte social del decir como conjunto de modalidades específicas de saber y de gobierno, describen cómo se integran determinados saberes, estrategias y tácticas de poder como modalidades singulares de conducción de los otros a partir del privilegio social de específicas prácticas del decir enseñar-, del trasmitir y del ordenar -educary del controlar -de la gestión de aprendizajes y competencias-.

Las políticas docentes son la analítica de los fines y de los medios por los cuales se delimita, organiza y conduce una práctica social como especializada modalidad de gobierno de la población, de cómo modelos sociales de enseñanza, educación y control se establecen a partir de regímenes de reglas y de normas, de conductas y de comportamientos que autorizan ciertas modalidades de formación de sujetos a través de un tipo específico de docencia como singular forma de gobierno social.

Como instrumental de análisis, las políticas docentes exhiben las estrategias y las tácticas por las cuales sujetos e instancias pretenden imponer elaborados dispositivos de saber y de decir como modalidad de gobierno desde modelos religiosos, morales, sociales, económicos y de gestión.

Las políticas docentes tienen por tarea exhibir la docencia como invención política, son la descripción de la serie de prácticas sociales que conforman el resultado de la conformación de modalidades de trasmitir verdad desde las formas de enseñanza, de educación o de aprendizaje. Quizá por ello, para algunas instancias de gobierno y de la sociedad esta actividad social es central para el dominio de la sociedad (GLOBE; PORTER, 1980, p. 12; BID, 2002; McKINSEY y Cp ${ }^{3}$, 2007; OREALC; UNESCO; ECOSOC, 2011, p.7; entre otros autores).

\footnotetext{
${ }^{3}$ Mc Kinsey \& Company es una firma de consultoría global que sirve a las empresas, gobiernos y organizaciones no gubernamentales. http://www. mckinsey.com/about_us -Consultado: 25 noviembre de 2014.
} 
Como útil teórico metodológico, las políticas docentes describen las modalidades políticas que conforman la actividad singular de transmisión de los saberes hegemónicos en la sociedad como forma imperante del decir verdad y de someter poblaciones a formas determinadas de poder. Las políticas docentes son pues, el objeto, el instrumento y el medio de la descripción de prácticas sociales de la docencia como gobierno de sí y de los otros (FOUCAULT, 2009), son la máquina de la descripción de la serie de los ejercicios de gobierno que intentan imponer determinadas modelos sociales de saber, de pensar y de decir.

Designamos políticas docentes al análisis de las prácticas discursivas producidas por instancias como las agencias internacionales, los gobiernos, las organizaciones magisteriales, la sociedad civil, el sector empresarial, los docentes, etc. Describimos políticas docentes a como las instituciones sociales conforman sistemas jurídicos y normativos que pretenden gobernar la docencia, las maneras por las cuales los sujetos docentes se sienten subordinados o no, excluidos o no, de cómo resisten o reinventan otras formas docentes. En este sentido, las políticas docentes se corresponden al análisis político de las prácticas docentes, esto es, de la integración de discursos y de poderes al actuar como estrategias y tácticas que pretenden imponer una figura de la docencia como instrumento de gobierno ${ }^{4}$.

\footnotetext{
${ }^{4}$ Por gobierno comprendemos la "relación entre sujetos y la relación consigo mismo. En el primer sentido, el [gobierno] es un conjunto de acciones sobre acciones posibles. Trabaja sobre un campo de posibilidad en el que viene a inscribirse el comportamiento de los sujetos que actúan: incita, induce, desvía, facilita o dificulta, extiende o limita, hace más o menos probable, llevado al límite, obliga o impide absolutamente. Pero es siempre una manera de actuar sobre uno o varios sujetos actuantes, y ello, en tanto que actúan o son susceptibles de actuar. Una acción sobre acciones” (Foucault, 2010, p. 237)
}

El campo de las prácticas discursivas sobre las cuales se movilizan las discontinuidades políticas sobre los docentes está integrado por disciplinas como la teología, la filosofía, la medicina, la psicología, la economía, las tecnologías educativas, las práticas administrativas y de gestión, a partir de las cuales, la sociedad da forma a diferentes prácticas docentes que determinan modalidades singulares para el oficio del maestro de escuela.

Las políticas docentes son pues, la analítica de la conformación de racionalidades políticas que delimitan, formulan e implementan las diferentes prácticas de la docencia, de cómo se conforman sujetos en instrumentos, medios y gestores de modalidades sociales imperantes del saber, del pensar y del decir, ellas mostrarán también, que la práctica docente transcurre por múltiples discontinuidades, entre las cuales es posible producir otras formas de asimilar, atribuirse y de reinventar otras prácticas de devenir docencia.

\section{De políticas docentes}

Sueño con el intelectual destructor de
evidencias y universalismos, el que señala
en las inercias y las sujeciones del presente
los puntos débiles, las aperturas, las líneas
de fuerza, el que se desplaza incesantemente
y no sabe a ciencia cierta dónde estará ni
qué pensará mañana, pues está demasiado
atento al presente. (FOUCAULT, 2008, p.
44)

Nos alejamos de categorías que denominan la actividad docente como apostolado, profesión o profesionalismo (ENGUITA, 1998, 2004; NÓVOA, 2009, 2014; TARDIF, 2012; entre otros autores), ya que en ellas se ha aceptado que la relación social del profesor es de subalternatividad, al 
no constituirse socialmente ni como profesión liberal (WEBER, 2002, p. 233), o por la conformación de una profesión dependiente, al no contribuir de manera directa en la producción (MARX, 2002, p. 339). Interesan analíticas que permitan pensar "el juego de transformaciones específicas, cada una con sus condiciones, sus reglas, su nivel, ligadas entre sí según esquemas de dependencia" ${ }^{5}$ (FOUCAULT, 2010, p. 680).

De este modo, el análisis de las políticas docentes se refiere a las discontinuidades de formación de los distintos regímenes de la docencia, para el caso, de una práctica del saber, del pensar y del decir como modalidad de gobierno de la sociedad desde el periodo colonial latinoamericano, hasta los días de hoy, exhibidos en algunas de las figuras de prácticas docentes como las de maestrodoctrinante (COMENIO, 1998; LOYOLA, 1491-1556; CALASANZ, 1557-1648; LA SALLE, 1651-1719); de preceptor-instructor de las ciencias (ROUSSEAU, 2000; LOCKE, 2012); de funcionario-educador (ENGUITA, 1998, 2004; TARDIF, 2012), de ciberantropo docente (FAURE, 1973; ZORA, 2015), de docente curricular (MARTÍNEZ, 1982), y de profesional de control (ZORA, 2015). Prácticas que conforman algunas de las distintas modalidades de la docencia en Colombia (ZORA, 2015).

\section{El maestro doctrinante}

Referimos como maestro doctrinante aquel sujeto formado por el conjunto de saberes religiosos desde el renacimiento hasta el siglo XVIII (FAVACHO, 2012; MARTÍNEZ, 1982). Considerando el periodo colonial

\footnotetext{
5 “o jogo de transformações específicas, cada uma com suas condições, suas regras, seu nível, ligadas entre si segundo esquemas de dependência” Trad. Luis F. Vásquez Zora.
}

latinoamericano, podríamos decir, que estuvo conformado por toda una serie de condiciones de existencia de saberes teológicos en torno de la práctica pastoral ${ }^{6}$ de reunir, evangelizar y doctrinar un rebaño, transformando por la palabra el niño o el infiel en conocedor de la verdad. El maestro proporcionó la disciplina, las reprensiones, los castigos o los cuidados necesarios a sus discípulos, esforzándose en revelar a Dios como único y verdadero maestro (AGUSTÍN, 1953), o haciendo de la escolástica la labor del maestro de enseñar por imitación de todo lo creado (TOMÁS, 2000) o sea, se trata de disciplinar el cuerpo como modalidad de cuidado de las almas que le eran encomendadas, enseñar es la práctica de evangelizar, de adoctrinar, de revelar un saber que reprenda el cuerpo a beneficio de una salvación más allá.

Ignacio de Loyola (1491-1556), José de Calasanz (1557-1648), Juan Bautista de la Salle (1651-1719), entre otros, conformaron con diferentes matices, instancias como son las escuelas religiosas, los colegios, seminarios, internados y casas para niños huérfanos y pobres, una serie de elaboraciones en torno del recogimiento del maestro frente a la doctrina y la defensa de la fe, como compendio orientador de un saber absoluto,

\footnotetext{
${ }^{6}$ Sobre la práctica del "poder pastoral” ver: Foucault, M. El sujeto y el poder. En: Dreyfus \& Rabinow. Michel Foucault. Más allá del estructuralismo y la hermenéutica. Ed. Nueva visión Buenos Aires. 2001. “1) Es una forma de poder cuyo objetivo último es asegurar la salvación individual en el más allá. 2) No es tan solo una forma de poder que dirige; se debe estar preparado para sacrificarse a él por la vida y la salvación del rebaño. 3) Es una forma de poder que se presenta no sólo ante toda la comunidad, sino ante cada individuo particular, durante toda su vida. 4) Esta forma de poder no puede ser ejercida sin conocer el interior de la mente de las personas, sin explorar sus almas, sin hacerlas revelar sus más mínimos secretos. Esto implica un conocimiento de la conciencia y una habilidad para dirigirla” (p. 246).
} 
Dios, como figura superior y soberana, la cual debería 'formar' el maestro de escuela.

Esta figura de maestro doctrinante forjada en la disciplina pastoral (FOUCAULT, 1998, 2001, 2004, 2006) por la conducta y el comportamiento guiado por hábitos ascéticos, por la distribución de horarios para cada una de las actividades diarias de acuerdo a las oraciones y los rezos como forma de distanciamiento del hombre de su estado natural, de sus materialidades, maestro como revelador de la divinidad como verdad al alma y disciplinador del cuerpo, doctrinador de toda uma ars divina como moral colectiva (MARTÍNEZ, 1982).

Corresponde a la docencia responder a las imposiciones exigidas por la moral de una vocación cristiana y al maestro ejercer el papel de discípulo, enseñar a Dios y sus virtudes, el cuidado y la contemplación del alma, el castigo y la pena para el cuerpo, lugar y origen del pecado. Su práctica de enseñanza se resume en una didáctica del maestro como poseedor de una episteme divina. Figura a través de la cual, la disciplina cristiana enseña al alma para la lucha contra el dominio del cuerpo y sus pasiones. También una función de policía moral (MARTÍNEZ et al. 2014, p. 54), de las poblaciones indígenas, negras y pobres; maestro-doctrinante que basa y reproduce prácticas cristianas de cuidado de sí, como los ejercicios espirituales, liturgia de las horas, los sermones, la enseñanza del catecismo, las oraciones, los misales y rezos (TOMÁS, 2000) que no serían más que hábitos regulares que buscaban la disciplinarización como conjunto de prácticas por medio de las cuales se dociliza al maestro, a quien "no solo le será necesario conocerse a sí mismo, sino, conocer para dominar a los demás” (COMENIO, 1998, p. 17).

De la práctica de la enseñanza de Dios como gobierno de si y del otro, la docencia toma las conductas cristianas de renuncia a sí por la obediencia al otro superior, "transsubjetivación” fue denominado por Foucault (2006, p. 263) a la práctica de invertir la propia subjetividad a partir de práticas de obediencia, de resignación y recogimiento a la voluntad de otro, asumido como superior y autoridad, frente al cual se es deudor de obediencia y humildad, y frente a si, renuncia, vigilancia permanente de los pensamientos, de examen de consciencia, de prohibición frente a los más mínimos pensamientos, sentimientos, deseos y actos perturbadores de la relación con la pureza y la verdad divina. La práctica del maestro-doctrinante basa su éxito en la enseñanza de la salvación, en el apagamiento del cuerpo y sus pasiones por los ejercicios de la disciplina del cuerpo a través de la rutina espiritual.

Las herramientas que debería posibilitar el maestro doctrinante para el gobierno de si y de los otros vendrían dadas por la doctrina, como modalidad de enseñanza, no solo de las primeras letras y cálculos, sino como figura de gobierno pastoral, debía cumplir el propósito de llevar la luz, la verdad, el conocimiento a la naturaleza humana obscura y salvaje, movida por los deseos y las pasiones. Tendría por función transmitir la luz de Dios a los sin luz, a los a-lunos, decir y revelar su palabra, enseñar para la salvación por medio del conocimiento de la divinidad. El maestro se opone, por lo tanto, a las materialidades del mundo, conocer es llevar la luz espiritual, como doctrinador, su función es libertar el espíritu de la prisión del cuerpo y la carne.

La doctrina como 'pedagogía', como trasmisión de una verdad que tiene por función dotar al sujeto de capacidad de renuncia así, por el conocimiento de los saberes teológicos propuestos como fin para la salvación. Como enseñanza doctrinal, la disciplina es la práctica de la docencia para la conversión de las almas, de renuncia de sí 
por la verdad, del disciplinamiento corporal (MARÍN, 2013). El maestro doctrinante, en tanto rebela, enseña y transforma el alma del otro, del infiel, del indígena, del negro, del pobre y del infante, tratados como ausentes de luz y de la verdad de Dios.

El maestro reúne y conduce, pastoreándose a sí mismo por el camino del bien divino, insiere un alma cristiana en aquellos que carezcan de ella. Guía como a un rebaño, renuncia a sí mismo por el amor a sus ovejas, por el conocimiento eterno y divino que les perfeccionaría, la práctica de la enseñanza de la doctrina, es pues, un arte de gobierno pastoral de sí y de los otros.

El maestro doctrinante es ejemplo de disciplina de sí, para disciplinar los otros, su didáctica, la enseñanza de la moral definida por la fe "docente padre ou sacerdote" (FAVACHO, 2010, p. 76). Durante el período colonial fue, pues, el medio de imitación del saber cristiano y del poder monárquico, doctrinador de las almas y disciplinador de los cuerpos, quien los prepara para obedecer a Dios y a la orden social impuesta por el monarca.

La práctica del que sabe y dice es, por lo tanto, herramienta política, dispositivo de disciplinamiento moral y de reprensión cristiana sobre el cuerpo. Conforme anunció San Agustín en el libro de Civitate Dei (413425 D.C.), el pastor, aseguraría la conducta espiritual para lo eterno, así como, el orden material deseado por Dios y por el monarca para sus súbditos en la tierra.

\section{El maestro preceptor}

El siglo XVIII hace emerger el hombre como centro de nueva positividad, como principio de ciencia y razón. El fondo blanco de lo eterno es restituido por la nueva orden de la historia natural, de la gramática y del libre cambio (FOUCAULT, 1968). Para el Iluminismo, el maestro es el mediador de la civilización, portador de la ciencia y de una verdad renovada, su función no será más la de propagar una enseñanza como moral divina, más si, un saber y una verdad apoyado en la comprobación de un método verídico: la nueva ley científica. En la ilustración es el preceptor de los conocimientos y de las leyes científicas, aquel que enseña para la vida en sociedad del ser natural y humano conforme al nuevo contrato social (ROUSSEAU, 2000).

El docente se torna en el sujeto que docta, que dice y transmite verdad a partir del reconocimiento de las leyes de la naturaleza, su hacer será ahora instruir al hombre en las cualidades y virtudes de la naturaleza, regresar al hombre al estado de bondad dado por la naturaleza al momento de nacer y que podrá ser restablecido por la educación a través del saber y el conocimiento de las ciencias: "todo lo que le falta al hombre al nacer, le será dado por la educación” (ROUSSEAU, 2000, p. 9). El maestro-preceptor prepara al hombre, al ser más desvalido que cuantos existen en la naturaleza, para que éste adquiera las fuerzas suficientes para enfrentar el mundo social.

El preceptor como maestro ilustrado instruye al hombre, ser sensible y natural en las estrategias del mundo social. Maestro preceptor, no más de todo cuanto existe y divino, más si, de las relaciones entre los hombres, de sus posibilidades para desarrollar habilidades, instruir al hombre para que sea ciudadano. Instruir sobre el saber natural, para que civilizado sepa vivir en sociedad. Así, es tornado en depósito del saber, "biblioteca viviente, cuerpo enseñante del pedagogo" (SERRES, 2012, p. 10).

Rousseau en el Discurso Sobre el origen y los Fundamentos de la Desigualdad entre los Hombres (1754), hace emerger el mito del buen salvaje, del estado de naturaleza, de las 
comunidades vírgenes y primitivas, del grado cero de virtud y bondad de la civilización y de la cultura. Quizá en aquel espacio, nosotros latino-americanos perdidos en el tiempo de lo recién hallado extenderíamos nuestras formas barrocas coloniales latinoamericanas bajo el deseo de una modernidad que no llega y de un modernismo que nos abruma, misturamos lo nuevo y lo antiguo, la escuela y la iglesia, el maestro doctrinante y el preceptor (MARTÍNEZ, 1982).

En América Latina, el preceptor se encontraba entre dos fuerzas: una de carácter religioso y otra de carácter ilustrado, mixtura el hacer de doctrinante religioso con la de transmisor de la ciencia. Este sincretismo entre la enseñanza religiosa y la instrucción del saber laico fue una de las formas de aparición de la instrucción como moral pública cristiana y no solo como enseñanza clerical ni como práctica ilustrada.

La instrucción se tornaría así en el ideal social, los estados regios la reclamaron para formar caballeros útiles al rey y a Dios (LOCKE, 2012). En nuestro continente americano, la expulsión de los jesuitas -el año 1759 en Brasil y el año 1767 en Colombia-, forzaría la aparición de las escuelas de primeras letras, laicas y públicas, renovadas reglas y vigilancias le serán impuestas al maestro preceptor de los estados monárquicos y coloniales: "quien se ocupase de este oficio tendrá que demostrar legitimidad, limpieza de sangre y buena conducta” (MARTÍNEZ, 1982, p. 81). El docente debería ahora someterse a la demonstración de todo um conjunto de características que comprobasen una moral cristiana, una vida ciudadana y pública virtuosa.

El maestro preceptor tendría por función la propagación de la episteme de las ciencias naturales, fue instructor, acompañante privado de los procesos de enseñanza, instruía los alumnos en las expresiones de la ley y de la verdad de los fenómenos científicos de la naturaleza y de la sociedad (ROUSSEAU, 2000). Esta figura del maestro preceptor de la ciencia y de la civilización se opone al primitivo, al no civilizado, al salvaje y al carente de la luz de la verdad científica.

\section{El maestro instructor moral}

Otro tipo de discurso y de práctica social de la docencia serán conformados en el siglo XIX. Los saberes de las ciencias aplicadas como la medicina, las ingenierías y la sociología aportaron profundidad a la superficie de saberes naturales clareados por la Ilustración. Los Estados republicanos van a disputar con la iglesia la hegemonía de la instrucción pública, fueron instituidos los primeros sistemas nacionales de instrucción y definidos los currículos, la escuela se torna institución pública bajo el dominio del Estado.

El maestro de escuela emerge como figura sincretizada por las modalidades del maestro doctrinante y del preceptor, al cual le sería asignado una renovada función pública, un nuevo papel como agente del progreso social, su función es ahora, la instrucción y la moralización de los ciudadanos del Estado.

En la primera mitad del siglo XIX los saberes positivos penetraron la escuela en Colombia, a través de la enseñanza lancasteriana, estrategia republicana para la alfabetización de la población que utilizó como maestros iniciales a los alumnos más adelantados del salón (ZULUAGA, 1984; OCAMPO, 1983). En la segunda mitad del siglo XIX, la enseñanza instruccional en el país consistía en "formar hombres saludables de cuerpo y de espíritu, dignos y capaces de ser ciudadanos y magistrados de una sociedad republicana y libre” (MALTE, 2012, p. 60). 
La enseñanza se tornaba útil como medida del progreso social, a la práctica del maestro le es exigida por la sociedad burguesa contribuir a la formación de cuerpos saludables y vigorosos, reforzar el objetivo de aumentar las ganancias de la industria, la riqueza y el poder del Estado. En este espacio fue producida una renovación en la docencia como función pública, entre maestros y alumnos, el Estado organiza la escuela, como también conforma sus medios disciplinares, los exámenes, la observación sobre docentes y alumnos, desarrolló una clínica (FOUCAULT, 2004) escolar, la inspección y el examen, a través de la cual va a dirigir su mirada sobre el disciplinamiento de los cuerpos para el trabajo y la producción.

La medicina, la economía y la sociología harían parte de la conformación de esa nueva episteme burguesa basada en el progreso, estas disciplinas localizan en el instructor moral, la figura entre el cuerpo individual y el cuerpo social, el disciplinamiento como tecnología política de normalización, establecen patrones, medidas, así como conductas biológicas y sociales, modalidades de inspección y vigilancia como modalidad de gobierno de la docencia y de las poblaciones.

De las ciencias médicas tomaría el examen, realiza pruebas, vigila el desarrollo físico de los niños, evalúa su alimentación, su vestuario y la higiene de los cuerpos. De la economía, la instrucción para el trabajo, valor de cambio más importante que el dinero en las sociedades burguesas, forma artesanos, operarios, comerciantes, profesionales, etc., se establecen las casas de recogimiento para niños pobres y huérfanos. De la sociología enseña que el orden entre el individuo y la sociedad es la condición normativa y necesaria para el progreso social, del cual, el maestro de escuela es medio de trasmisión y permanencia del ordenamiento de la moral social.
En el maestro instructor moral confluyen toda una ars social disciplinaria para la normalización de sí y de los otros, como bandera del Estado, es pieza y agente para la modernización social, ejemplificador del orden para el progreso (ZULUAGA, 1987). Maestro de escuela, instructor de la moral social que debe poseer los individuos con la finalidad de superar los desafíos de la modernización, su función contribuir en la prevención de todo aquello que tenga relación directa con la anormalidad, la perversión, la insanidad, la pobreza y la ignorancia. El maestro de escuela pública se torna, por lo tanto, en instrumento de normalización y moralización para normalizar y moralizar otros.

Todas estas modificaciones hacen parte de una serie más amplia de lógicas de dominación que pretendieron hacer de la instrucción pública nacional elemento político del orden social y del maestro agente e instrumento socializador. Como práctica de instrucción y de moral del Estado, las instancias involucradas -iglesia, gobiernos, instituciones escolares de enseñanza elemental, escuelas normales, corporaciones, etc., de un ordenamiento jurídico, basado en el derecho natural y en las libertades fundamentales, de la fundación de escuelas nacionales, de institutos de artes y oficios, de bibliotecas nacionales, museos, observatorios, archivos, etc. -, la actividad del maestro fue propuesta como sofisticado dispositivo de tránsito entre órdenes y estrategias sociales que involucran "el individuo, la familia, las corporaciones, el Estado y la iglesia, así como el consorcio entre las Municipalidades, los Departamentos y la Nación, para trabajar de consuno en la obra múltiple y fecunda de la instrucción y la moral del pueblo" (URIBE, 1927, p. VIII).

En la reorganización de la Educación Nacional (Conformación del Ministerio de 
Educación Nacional, Colombia. Ley n. 56 de 1927), la instrucción y la moral católica se mixturan: "preparar a los niños para el ejercicio de la ciudadanía, despertando y avivando en ellos el amor a la patria; prepararlos para la agricultura, la industria fabril y el comercio” (URIBE, 1927, p. VII). Frente a las prácticas religiosas se afirmó que la religión es el "alma y la identidad nacional” y la instrucción de la nación debe "procurar que por las enseñanzas religiosas católicas se formen espíritus de carácter noble; hombres de energía, saludables y vigorosos” (URIBE, 1927, p. VII).

El maestro de escuela es, pues, instructor moral, exhibe una práctica docente producto de un gobierno que tiene como guía de disciplinamiento la moral cristiana católica y un conjunto de conocimientos de disciplinas prácticas para la explotación de los recursos de la nación. Las políticas docentes tienen como práctica la instrucción moral, esto es, comprende las funciones sociales del gobierno de la vida, de los cuerpos vigorosos para la producción, de la conducción del alma y de la moral como economía de los hombres.

La sociedad del siglo XIX y de comienzos del XX arrastra sobre si la transición de la soberanía como relación de poder frente a la extensión de un territorio, el dominio de sus recursos y la conformación de poderes públicos que emanan de los que en él habitan. En este período en América Latina estuvo constituida por naciones conservadoras, tradicionalistas, católicas y con pretensión de positividad.

La aparición del maestro de escuela pública obedece a la emergencia de un sujeto poco visible, funcionario asimilado a los demás empleos de la institución escolar, sujeto forjado para la construcción de obediencias entre las instancias clericales, las asociaciones mutuales y la nación: “el espíritu católico y conservador sirva de vivificador de la experiencia, no aceptando innovaciones que tienen el carácter de revolución, aunque espíritus brillantes señalen la civilización y el progreso que vieron en otras latitudes; pero ignoran el viacrucis por donde nosotros transitamos” (RESTREPO, 1936, p. 176).

En este maestro instructor moral confluyen elementos de una ocupación de carácter religioso y laico, caracterizado por la caridad, por el valor y por la entrega voluntaria al otro como práctica religiosa, cristiana y disciplinaria, pero también, filantrópica, humanista y normalizadora, una práctica de escuelas normales a cargo de los hermanos cristianos en la conformación de un oficio caracterizado por la vocación, la obediencia pastoral, la conservación de la tradición, del orden patrio y civil. En la figura del maestro instructor moral confluye al mismo tiempo, la práctica cristiana de instruir, transformar y conducir al otro por la orden divina, la moral y las leyes del Estado republicano, como modalidades políticas del maestro para el progreso y la moral de la nación.

\section{El maestro medio: entre el individuo y la sociedad}

En la primera mitad del siglo XX los sistemas educativos en Colombia fueron renovados a partir de dos elementos principales: la reorganización administrativa de los sistemas nacionales de instrucción pública y la pedagogía experimental como exigencia de soporte epistémico para la educación. En 1934 la Oficina Internacional de Educación OIE realiza la primera conferencia mundial recomendando a las naciones que éstas “deben reorganizar sus sistemas nacionales de instrucción pública y definir la pedagogía experimental como base 
del ejercicio de la docencia” (UNESCO, 1979, p. XIII).

Esta reorganización administrativa y pedagógica hace parte de un conjunto de recomendaciones internacionales conocidas como la "reorganización de los sistemas de instrucción pública entre los años 1934 y 1939” (UNESCO, 1979, p. XII), en ellas, los maestros fueron designados por primera vez, como "personal docente" (OIE, 1934, p. 5). La Sociedad de Naciones exigió a los Estados a través de la Oficina Internacional de Educação OIE, la reorganización del personal docente en procesos de: "reclutamiento, formación, perfeccionamiento, cuadros técnico-científicos, estatutos y remuneración" (OIE, 1935, p. 5). Aquello torna la docencia en medio de las relaciones entre sociedad e individuo, entre economía y educación, la práctica docente tendría a partir de entonces, la función de mediar entre el sí y los individuos, llevando a cabo los objetivos que la sociedad establece para la educación como principio de gobierno, la docencia es práctica que mediará entre la formación humana y una nueva administración económica y moral de los sujetos.

Como medio contribuyó para la capacitación de la población en técnicas y oficios, el docente como mediador deberá llevar adelante la educación de los ciudadanos para el crecimiento económico y la socialización de la población. El maestro adquiere, por lo tanto, un nuevo papel en las relaciones económicas, al ser designado como 'personal docente' pasa a ser, simultáneamente, recurso de trabajo y formador de capacidades económicas en la población.

Más que reconocer el oficio docente en la primera mitad de siglo XX, a partir de la categoría trabajador, bajo la relación directa o no con la producción social, o como transmisor o no de una ideología de clase, se trata aquí de describir las condiciones de existencia de prácticas políticas docentes: sujeto trabajador y capacitador para que otros trabajen, de lo cual la OIE expresaba: "el reclutamiento tiene que ser de personas escogidas, ya que les son confiadas el porvenir de la nación" (OIE, 1934, p. 6). Como si la docencia fuese la práctica social de importancia para obtener los objetivos económicos y sociales, para el alcance del bienestar y el porvenir deseado por las naciones y a su vez, medio de regulación de la vida económica de los individuos y de las poblaciones.

El segundo elemento de reorganización educacional en este período consistió en la definición de la pedagogía experimental como base epistémica del ejercicio docente. Por pedagogía experimental se comprendió la “formación psicológica integrada en la preparación pedagógica general y en particular en su preparación de la didáctica” (UNESCO, 1979, p. 29). En las Recomendaciones $N^{\circ} 4$. De la formación del personal de enseñanza básica (1935) y $\mathrm{N}^{\circ}$ 5. De la formación del personal de segunda enseñanza (1935), de la OIE, la pedagogía experimental constituye el núcleo común para la formación y debe tornarse en ejercicio práctico de los docentes, en otras palabras, la formación disciplinar de los docentes sería establecida por la pedagogía experimental, como condición de formación y como nuevo saber y práctica de la docencia.

Para el discurso pedagógico experimental las orientaciones internacionales conducen el sujeto y su práctica por una positividad comprobable y científica. La orden epistémica definía la base positiva experimental -la psicología de la infancia, biología y medicina-, así como las modificaciones en el sujeto y sus prácticas a partir de la reorganización administrativa de los sistemas nacionales de instrucción pública y de los haceres docentes. 
En este período lo que acontece no es más que la reorganización de conceptos, de sujetos y de sus prácticas bajo una positividad pedagógica basada en el carácter experimental, reorganización de la docencia como expresión estratégica de las 'Recomendaciones Internacionales sobre la instrucción pública’, como práctica discursiva, la pedagogía experimental presenta dos efectos políticos, uno horizontal, representado en las orientaciones de las organizaciones internacionales dirigidas a los gobiernos y la reorganización de sus sistemas educativos, y otro vertical, sobre las prácticas docentes, definiendo su hacer a partir de metodologías pedagógicas experimentales como fundamentos de la formación, carrera y ocupación que tienen como base los fundamentos experimentales de la enseñanza.

Los movimientos estratégicos de las ciencias aplicadas, lentamente conformaron la pedagogía experimental en un tipo de práctica que por un lado, propuso un contenido de saber comprobable, por otro, un conjunto de acciones políticas de carácter administrativo que conformarían la docencia en hacer práctico experimental.

Por reorganización de la educación se refiere a la relación entre la sociedad y el individuo, entre el grupo y la unidad, entre el órgano y el cuerpo social, reorganizar la educación, involucró, por lo tanto, relaciones sociales entre educación, docencia y economía, o sea, entre la episteme pedagógica experimental, la categorización de la docencia como oficio social y los desafíos sociales y económicos del Estado. Lo que significaba no solamente modificaciones en los sistemas de instrucción pública con base en una pedagogía científica, sino también, medidas económicas de la nación en la designación de la docencia como oficio social, en las mudanzas de los conceptos pedagógicos, así como modificaciones en las prácticas de los sujetos maestros de escuela.

Reorganización educacional docente comprende la reagrupación de los docentes bajo la categoría del oficio, su reconocimiento y reafirmación como trabajador con algunas herramientas conceptuales y metodológicas experimentales ofrecidas por la instrucción como técnica educativa que reorganiza la positividad de su hacer. Asistimos, entonces, al inicio de una docencia organizacional en cuanto se propone la reorganización de su positividad a partir de las práticas experimentales, modificando a su vez, la labor moral de la patria por una función docencia de carácter experimental y productiva.

\section{Del Ciber-Antropo docente}

Las recomendaciones internacionales de instrucción pública en los años de 1934 a 1939 propusieron la reorganización de los sistemas educativos en grupos y unidades educacionales, ya en la segunda mitad del siglo XX fue propuesta la restructuración de los sistemas educacionales. La “reestructuración de la Educación” propuesta por la OIT-Unesco (1966) comprendida por una mejor distribución y disposición de los docentes en la estructura social, la docencia no es más nombrada como oficio, ahora lo será como profesión. La "Recomendación relativa a la situación del personal docente" (OIT-UNESCO, 1966, p. 8), estableció las "normatividades internacionales sobre la profesión docente” o el reconocimiento de la docencia como ocupación perteneciente a una "profesión de tipo social, ético, y material relativa a los docentes” (OIT-UNESCO, 1966, p. 2).

Los parámetros que definen la docencia son la capacitación, la contratación, la carrera, la progresión, la seguridad en el empleo, los 
procedimientos disciplinares, el servicio de tiempo parcial, la libertad profesional, la supervisión, la evaluación, la participación en la toma de decisiones en materia educativa, la negociación, las condiciones favorables para la eficacia de la enseñanza y el aprendizaje (OIT-UNESCO, 1966). Conformando de este modo, todo um conjunto sistematizado de elementos, una estructura definida desde las prácticas discursivas de carácter administrativo y pedagógico que delimitan la docencia en profesión.

Con estas formulaciones el Estado intenta llevar a cabo uno de sus propósitos, la "constitución de un sistema sobre los maestros" (OIT-UNESCO, 1966:), o sea, la reestructuración de la docencia como agente activo para el desarrollo: la formación de un docente crítico y transformador, durante las décadas de 1960 y 1970 el maestro de escuela, es "potencial docente, el capital humano clave para el desarrollo socio-económico de la América Latina” (MURILLO, 2005, p. 7), pero también, es mucho más, es propuesto como maestro técnico y científico que se apropie de las herramientas de las tecnologías educativas para la formación de un "hombre completo, científico, técnico y ciudadano: un Ciber-antropo docente" (FAURE et al., 1973, p. 7). El maestro de escuela debería ahora “acompañar la evolución social y política, la evolución científica, técnica y económica (FAURE et al., 1973, p. 28).

Sin embargo, este ciber-antropo docente, transformador, crítico, tecnocientífico, no sería más que el resultado de la confluencia de los discursos de la ingeniería, de la administración, de la tecnología, etc., de los cuales, las políticas públicas como saberes aplicados buscarían conformar de modo que pudiesen intervenir en la estructura social, enfrentando el desafío de producir el hombre técnico-científico de la sociedad moderna que pretendía constituirse y afirmarse como modernizada, alejando así, nuestros países latino-americanos de la idea del atraso que representa el subdesarrollo, la ignorancia y la pobreza.

Un sujeto ciber-antropo docente como apoyo al esfuerzo social de potencializar la ciencia, la tecnología, la democracia y la cultura, el cual tendría entre sus funciones coadyuvar a la formación de las dimensiones humanas, técnico-científicas, económicas y ciudadanas. Estas serían una de las condiciones de surgimiento del texto aprender a ser (FAURE, 1973), las de un docente como hombre completo, científico, técnico, promotor del desarrollo, una práctica docente de ciber-antropo.

\section{Del maestro cultural, profesional y de competencias}

Para finales de la década de 1980 y mediados de 1990 algunas modificaciones acontecen en la profesión: "aspectos culturales, raciales o étnicos, relaciones de género y cuestiones de la subjetividad” (MANCEBO, 2007, p. 467), hicieron la profesión girar en torno de la idea de una docencia como acción cultural. La importancia de una actividad más centrada en el aprendizaje, entre la relación del pensamiento y la creatividad, en busca de nuevos valores, de nuevas acciones y reflexiones sobre el carácter democrático, inclusivo, participativo e internacional, una práctica que se dirigiese al encuentro de un hombre integral como nueva lógica de formación de sí y de los otros, "el conjunto de las acciones de la docencia deben orientarse hacia el aprendizaje, deben dirigirse hacia la formación de un hombre inacabado" (UNESCO, 1990, p. 158).

Las políticas docentes estarían, entonces, sobre el paso de la reforma de la profesión a la profesionalización. La 
reforma de la profesión docente no buscaba reformar la enseñanza ni la educación, pero si, el aprendizaje como "el nível de los conocimientos y las informaciones para mejorar la calidad de la vida, es aprender a aprender” (UNESCO, 1990, p. 2). “Aprender a aprender” definido en "cuatro contenidos fundamentales: aprender a conocer, aprender a hacer, aprender a vivir juntos y aprender a ser" (UNESCO, 1996, p. 47). Aprender desde entonces, será conocer lo otro y los otros para poder gobernarse así mismo.

Como agente profesional de la cultura, como generador de conocimientos, de haceres, de aprendizajes, de vidas comunes con fines propios, fundara su práctica en la inclusión, en las aptitudes, en las posibilidades, habilidades y destrezas, esto es, en la pedagogía de las competencias, definiendo por estas últimas "los conocimientos, la calidad profesional y la motivación de la diferencia y la inclusión” (UNESCO, 1996, p. 81).

El docente como agente cultural orientado por la adquisición de aprendizajes en un "mundo en permanente crecimiento económico y comercial, de libertades políticas y diversos valores culturales, [lo cual] el docente debería ayudar a ampliar las diversas opciones humanas” (PNUD, 1995, p. 15). Este sujeto maestro, expectativa de múltiples reformas en los años de 1990, -el cual continua siendo objeto de reformas-, pasa a ser el agente forjado para estimular las diferencias de un mundo de interdependencias, de fronteras económicas y financieras abiertas, de un proyecto global que busca apaciguar las diferencias políticas y culturales.

En Colombia el Movimiento pedagógico (FECODE, 1982), propuso como práctica una docencia desde la experiencia cultural, esto es, como práctica pedagógica ${ }^{7}$, como

${ }^{7}$ Sobre el concepto de práctica pedagógica V. Zuluaga, 1987; Zuluaga y otros, 2003. Además del Grupo de elaboradora de saberes propios y en contexto, maestros sujetos de saber y agentes culturales que propusiesen sus prácticas desde la resignificación epistemológica de la actividad (RODRÍGUEZ, 2009, p. 7; TAMAYO, 2006, p. 102; ZULUAGA, 1987, p. 8), en la cual los docentes fueran actores sociales y colectivos que darían "mayor sentido a su quehacer" (MEJÍA, 2006, p. 290).

Este Movimiento pedagógico, "lanzó a los maestros a las puertas de las facultades de educación, no como objeto sino como potencia, como capacidad de ser afectado por muchas relaciones de la más diversa índole que le dan a conocer su potencia y límites” (ZULUAGA, 2002, p. 314); al proponer también: “1. La producción de un saber específico; 2. Relación con otros saberes; 3. Relación con el hombre público y 4. Relaciones con su deseo y cotidianidad” (p. 314).

El papel cultural del educador implicaba que los maestros asumiesen la práctica pedagógica como desafío epistemológico y estrategia de resignificación de la labor frente a la sociedad y los gobiernos, esto es, la “definición de conceptos ‘borrosos' y poco claros como el propio concepto de pedagogía, pues se trataba de rescatar el carácter básico y fundamental de la pedagogía como practica propia del maestro" (ZULUAGA, 1987, p. 8), rescate epistemológico de la docencia a partir del concepto de práctica pedagógica que además de oponerse a la instrumentalización de la enseñanza, enfrentó de manera crítica las reformas de las políticas educativas, lo cual significó que los docentes se tornaron en agenciadores de saber, de un quehacer con límites epistemológicos precisos, con herramientas teórico-pedagógicas propias y demarcación de la práctica pedagógica

Historia de la Práctica Pedagógica en Colombia: http:// historiadelapracticapedagogica.com/ 
como lugar natural para la expresión del sí y del hacer docente, de sus relaciones con los saberes y disciplinas específicas, y con la sociedad.

Sin embargo, para los poderes que se pretenden imperantes, -agencias internacionales, gobiernos, sociedad civil, corporaciones, etc. -, el modelo de docente agenciador, gestor, inclusivo, controlador y estimulador de los padrones para la mundialización, con la función pedagógica otorgada sobre los aprendizajes, pretende la adquisición de una toma de consciencia generalizada de un "docente que oriente en la búsqueda de soluciones en un mundo exterior lleno de múltiples y complejos problemas" (UNESCO, 1990, p. 18), esto es, la conformación de un sujeto de control, agente y gestor para un modelo social basado en un mundo en competencias, una práctica que estimule la producción de otra modalidad de gobierno de sí y de los otros como población de la gestión de los aprendizajes, de las habilidades y de las destrezas, ya no se trata de la relación maestro-discípulo, se trata de conocer lo otro y los otros, para poderse competir consigo mismo.

El maestro de las competencias delimita la práctica de la docencia a la evaluación de desempeños, a los incentivos, a la innovación y a la excelencia, no se trata más de la experiencia ni de la profesión como medida de carrera, ascenso y ganancias individuales y colectivas, sino de la gestión por objetivos, por incentivos, por la obtención de premios, de productivismos, de salario por desempeño y resultados, sea a través de técnicas evaluativas que establecen estándares y medidas para una "docencia de excelencia” (MEN, 2015) según estándares y certificados de mercado establecidos para la escuela, la región escolar, el país, hasta conformar niveles de excelencia nacional e internacional que definan y certifiquen la calidad de la educación, así como las prácticas la excelencia como medida para la destinación del presupuesto y del financiamiento escolar y docente (MEN, 2014; UNESCO, 2015).

Nuevas políticas docentes son producidas a partir de estándares evaluativos nacionales e internacionales, una nueva racionalidad profesional a través de las prácticas que miden el desempeño por la evaluación de conocimientos pedagógicos de base, resultados de alumnos en pruebas nacionales, prácticas pedagógicas innovadoras y experiencias de aula estimuladas por los premios a las buenas prácticas pedagógicas, innovación en producción académica, materiales didácticos, investigaciones, percepción y evaluación por parte de organizaciones de la sociedad civil y de padres de familia (COMPARTIR, 2014; MEN, 2015; PREAL, 2004). Todo un dispositivo social de gestión estratégica por competencias que no se limita a la evaluación como centro, ya no solo de la calidad, sino que le es agregado el desempeño que marche "tras la excelencia docente” (COMPARTIR, 2014), como práctica insaciable e infinita que asegure una sociedad de excelencia.

En Colombia la profesionalidad de los docentes aparece como pieza central de los sistemas educativos nacionales en la medida en que conforme a los docentes en agentes para el desarrollo de una política educativa de Estado, de "aseguramiento de la calidad de la educación a partir de la evaluación y el desempeño por competencias como medida o estándar de excelencia educativa, tanto nacional como internacional" (PARODY ${ }^{8}$, 2014). El programa del Ministerio de Educación Nacional: Excelencia docente (MEN, 2014), se orienta hacia esta dirección, pretender constituir al maestro en eje

${ }^{8}$ Gina Parody, Ministra de Educación Nacional de Colombia, a partir del mes de agosto de 2014. 
gubernamental de las políticas educativas bajo la consigna publicitaria de "a excelencia docente, excelencia educativa” (PARODY, 2014), lo cual no significa más que la hoja de ruta trazada por diversas agencias internacionales, organizaciones nacionales y de la sociedad civil, algunas de ellas, el "Pacto Nacional: todos por la Educación" $(2014)^{9}$ y principalmente, como eje de políticas educativas nacionales, el informe “Tras la excelencia docente. Cómo mejorar la calidad de la educación en Colombia” (COMPARTIR, 2014), que focalizan en la profesionalización de la docencia el núcleo político de mejoramiento de la calidad de la educación nacional, "la importancia de la excelencia docente para el mejoramiento de la calidad educativa detalla una ambiciosa propuesta sistémica de reforma educativa en Colombia que tiene como eje al docente” (COMPARTIR, 2014, p. 7).

Ahora, al docente le es exigido el control y la gestión de sus actividades escolares, que innove consigo mismo, haciendo de los otros innovadores, emprendedores y empresarios, una docencia que se gobierne menos gobernando más.

\section{Consideraciones finales}

La docencia es la sofisticada invención de prácticas históricas y políticas producidas

\footnotetext{
9 “El Pacto Nacional: Todos por la educación es un movimiento ciudadano que busca convertir la educación en una prioridad nacional. su objetivo es el desarrollo del talento humano para hacer de Colombia una nación justa, feliz y educada. Su trabajo se desarrolla en tres pilares: Promover la participación de la ciudadanía en la construcción de educación de calidad, facilitar el acceso a la información sobre la situación y el estado de la educación en Colombia y desarrollar proyectos de innovación social que busquen mejorar la educación del país”. http://www.todosporlaeducacion.co/colombia/ index.php/educacion/quienes-somos. Consultado: 10 mayo de 2015.
}

a partir del juego entre instancias y sujetos, entre órdenes de saber, estrategias y tácticas de poder con el intento de imponer determinadas docencias como especializadas modalidades de gobierno social. La docencia no es solo una 'cuestión de formación, de carrera y de remuneración’, es una práctica histórica y política discontinua, elaborada por la sofisticación de objetos, de medios e instrumentos para el gobierno de la población en una serie de historicidades sociales determinadas.

Con la descripción de algunas figuras políticas docentes exhibimos como ciertas prácticas discursivas y modalidades de poder, próximas o lejanas de la pedagogía, cercanas o distantes de la enseñanza, de la educación o del aprendizaje disponen saberes, fines y medios con la pretensión de producir efectos de gobierno. Las políticas docentes son, por lo tanto, especializadas tecnologías de poder por las cuales se reproducen modalidades de disciplinamiento, de normalización y de control del docente y de las poblaciones.

Si bien la sociedad pretende hacer imperar figuras políticas determinadas para el sujeto social que tiene como función la formación de sí mismo y la formación de otros, concebimos que en este juego, la docencia ocupa un lugar horizontal, con opción de poder reinventar otras prácticas: ¿No sería necesario que produjésemos otros tipos de docencia, distintas a las que estamos siendo? ¿Es posible conformar otras modalidades políticas para aquel sujeto que tiene por función el ver, el saber, el pensar y el decir? Quizá otras experiencias de la docencia puedan multiplicar las formas de devenir docente y por tanto de gobernarnos a nosotros mismos.

Ejemplos de reinvención de otras prácticas de la docencia lo conforma el Movimiento pedagógico colombiano 
(FECODE, 1982) y la Expedición pedagógica Nacional (MARTÍNEZ, 2002), como conjunto de acciones que propusieron "una mirada del docente sobre sí mismo” (TAMAYO, 1986, p. 48), incluyendo el rescate de su hacer, a partir de su práctica pedagógica y el involucramiento horizontal de los agentes pedagógicos nacionales -maestros, intelectuales, investigadores, universidades, organizaciones magisteriales, movimientos sociales, etc.-, como alternativa a la elaboración de un proyecto pedagógico educacional nacional, así como de búsqueda por establecer medios de acción frente al modelo cultural en disputa, frente a las políticas educativas nacionales y en oposición a la formulación de un docente instrumental y tecno-científico, y en su lugar convertir la práctica de la docencia en "una expedición, en una experiencia, a partir de una lectura múltiple del camino recorrido, de tal modo que esto nos constituya en productores de saber pedagógico" (MARTÍNEZ et al., 2002, p. 64).

Como descripción final digamos que le toca ahora al docente producir un giro en sus políticas, esto es, apropiarse, atribuirse y trasgredir su hacer en tanto conformación de otras prácticas de la docencia como devenir político posible.

\section{Referências}

AGUSTÍN, S. Del maestro. Biblioteca de autores cristianos. Madrid: Católica, 1953.

AMÓS, C. Didáctica magna. México, D.F: Porrúa, 1998.

AQUINO, S. T. De magistro: sobre o mestre. São Paulo: Unisal, 2000.

ARISTÓteles. Poética. Madrid: Gredos, 1999.

Retorica. Madrid: Gredos, 1999.
BOSCO, J. Escritos sobre el sistema preventivo en la educación de la juventud. Madrid: Biblioteca Nueva/Ministerio de Educación y Ciencia. (Serie Clásicos de la Educación, nº 7), 2003.

BOVET, P. El instinto luchador. Psicologíaeducación. Madrid: Biblioteca Nueva/ Ministerio de Educación y Ciencia. (Serie Clásicos de la Educación nº 19), 2007.

BID. Banco Interamericano de Desarrollo. ¿Quiénes son los maestros?: carreras e incentivos docentes en América Latina. Washington, DC: BID. NAVARRO, J. C. (Editor), 2002.

BONIFAZ, R. Ministerio de Educación: dirección de políticas pedagógicas plantea carrera docente ligada al mérito. Santiago de Chile: El Mercurio, 2014.

CALASANZ, J. 15 cartas a un colaborador laico. Madrid: Calasancias, 2007.

CLAPARÈDE, É. La educación funcional. Madrid: Biblioteca Nueva/MEC. (Serie Clásicos de la Educación nº 17), 2007.

COX, C. Nueva agenda de políticas docentes en América Latina y el Caribe. Buenos Aires: Unesco, 2011.

DECROLY, O. La función de globalización y la enseñanza y otros ensayos. Madrid: Biblioteca Nueva/Ministerio de Educación y Ciencia (Serie Clásicos de la Educación $n^{0}$ 13), 2006.

DELORS, J. La educación encierra un tesoro. Madrid: Ed. Santillana, 1996.

DEWEY, J. Mi credo pedagógico. Buenos Aires: Centro editor de américa latina, 1977.

DURKHEIM, E. Educación y sociología. Bogotá: Linotipo, 1979.

FERNÁNDEZ, M. Educar em tempos incertos. Porto Alegre: Artmed, 2004. 
La cara oculta de la escuela. Educación y trabajo en el capitalismo. Madrid: Siglo XXI, 1998.

FAURE, E. et al. Aprender a ser. la educación del futuro. Madrid/París: AlianzaUnesco, 1973.

FAVACHO, A. M. P. Rir das solenidades da origem: ou o inesperado da pesquisa em educação. Educação e Pesquisa, São Paulo, v. 36, n.2, p. 555-569, maio/ago, 2010.

FECODE. XII Congreso nacional de educadores. Conclusiones. Bogotá: Fecode, 1982.

FOUCAULT, M. Ditos \& escritos. Problematização do sujeito. Psicologia, psiquiatria e psicanálise. Rio de Janeiro: Forense universitária, 2010.

El gobierno de sí y de los otros: curso en el Collège de France (1982-1983). Buenos Aires: FCE, 2009.

Seguridad, territorio, población: curso en el College de France (1977-1978). Buenos Aires: FCE, 2006.

El nacimiento de la clínica. Buenos Aires: Alianza, 2004.

El sujeto y el poder. Posfácio. In: DREYFUS, H. L.; RABINOW, P. Michel Foucault. más allá del estructuralismo y la hermenéutica. Buenos Aires, Nueva visión, 2001.

Historia de la sexualidad I. la Voluntad de Saber. México. D.F: Siglo XXI, 1998

El orden del discurso. Buenos Aires: Letra E, 1992.

Arqueología del saber. México: Alianza, 1979.

Las palabras y las cosas. México:

Siglo XXI, 1968.
HAYEK, F. The constitution of liberty. London: The University of Chicago Press, 1978.

HIPONA, A. A cidade de Deus. São Paulo: Vozes, 2002.

KANT, I. Sobre pedagogía. Buenos Aires: Aleph, 2000.

LOCKE, J. Pensamientos sobre la educación. Madrid: Akal, 2012.

LOYOLA, I. Obras completas. Madrid: Católica. Biblioteca de autores Cristianos, 1977.

MANCEBO, D. Agenda de pesquisa e opções teórico-metodológicas nas investigações sobre trabalho docente. Educ. Soc., Campinas, vol.28, № 99, ago., p. 466482, 2007.

MALTE, R. El modelo pedagógico de la reforma instruccionista de 1870 en el Estado soberano de Santander: un modelo para la formación de ciudadanos. Revista Historia 2.0, Conocimiento histórico en clave digital. $\mathrm{N}^{\circ}$ 2. Ed. Universidad Industrial de Santander, agosto de 2011-marzo-2012.

MARTÍNEZ, A. Escuela, maestro y métodos en Colombia (1750-1820). Bogotá: UPN, 1985.

. La aparición histórica del maestro y la instrucción pública en Colombia. Bogotá: UPN, 1982.

MARTÍNEZ, A.; NOGUERA, C; CASTRO, J. Pedagogía, enseñanza de las ciencias y modelo curricular. Revista de Educación y Cultura. Bogotá, Fecode, n. 17, marzo, 1989.

MARTÍNEZ, A.; UNDA, P; MEJÍA M. El itinerario del maestro: de portador a productor de saber pedagógico. In: SUÁREZ, H. Veinte años del movimiento 
pedagógico. Bogotá: Cooperativa editorial magisterio, 2002.

MARTÍNEZ, A. et al. Escuela pública y maestro en América Latina. Historias de un acontecimiento, siglos XVIII-XIX. Buenos Aires: Prometeo libros, 2014.

MARX, K. El capital. Libro I. México: Porrúa, 2002.

MARX, K.; ENGELS, F. El manifiesto comunista. México: Porrúa, 1978.

MEJÍA, M. Educación(es) en la(s) globalización (es). Bogotá: Desde Abajo, 2012.

MIGUEL, R. Nuevo diccionario latino español etimológico. Madrid: Visor, 2001.

MONTESSORI, M. El descubrimiento de la infancia: método de la pedagogía científica. Madrid: Serie Clásicos de la Educación, nº 6, 2003.

MURILLO, M. Sindicatos, coaliciones partidarias y reformas de mercado em América Latina. Madrid: Siglo XXI, 2005.

NOVOA, A. Diálogos de saberes na prática profissional docente. Palestra. FaE-UFMG. 12/03/2014.

Professores: Imagens do futuro presente. Lisboa: Educa, 2009.

OCAMPO, J. Educación, humanismo y ciencia. Historia de las ideas fundamentales en el desarrollo de la Universidad Pedagógica y Tecnológica de Colombia. Tunja: UPTC. Ed. La rana y el águila, 1983.

OIE. Recomendación Conferencia Mundial de Instrucción Pública. $N^{\circ} 5$. De la formación del personal de segunda enseñanza. Ginebra, 1935.

Recomendación Conferencia Mundial de Instrucción Pública. $N^{\circ} 3$.
Las economías en la esfera de la instrucción pública. Ginebra, 1934.

OIT-UNESCO. Recomendación conjunta de la OIT y la UNESCO. Relativa a la situación del personal docente. París, 1989.

PESTALOZZI, J. Como Gertrudis enseña a sus hijos. México: Tipografía Antonio M. Rebolledo, 1889.

PIAGET, J. Psicología y pedagogía. Barcelona: Crítica, 2001.

PLATÓN. Las leyes. Madrid: Gredos, 1999. 1988.

La república. Madrid: Gredos,

. Protágoras. Madrid: Gredos, 1985.

PNUD. Informe sobre el desarrollo humano. México: Harla S. A. de C. V., 1995.

POL-DOIT, R. Entrevistas con Michel Foucault. Buenos Aires. Paidós, 2008.

PREAL. Maestros de escuelas básicas en América Latina: hacia una radiografía de la profesión. Santiago de Chile: San Marino, 2004.

QUINTILIANO, M. Instituciones oratorias. Tomo I. Madrid: Imprenta Perlado Páez \& Co., 1916.

RESTREPO, G. El pensamiento conservador: ensayos políticos. Medellín, 1936.

RODRÍGUEZ, R. El maestro en Colombia: hitos, avatares y paradojas. Bogotá: Universidad Distrital, 2009.

ROUSSEAU, J. Emilio o la educación. México: Alianza, 2000.

Discurso sobre a origem e os fundamentos da Desigualdade entre os homes. Lisboa: L\&PM Pocket, 1998.

SAÉNZ, J; SALDARRIAGA Ó.; OSPINA, A. Mirar la infancia: pedagogía moral y 
modernidad en Colombia. (1903-1946). Medellín: Universidad de Antioquia, 1997.

SALLE, J. Obras Completas de San Juan Bautista de La Salle. Obras pedagógicas y escolares. Madrid: Hermanos de las escuelas cristianas España, Portugal y América Latina Lasallista, 2001.

SCHERPING, G. Formación, evaluación y desarrollo profesional de los docentes: ejes clave de las políticas educativas latinoamericanas. Santiago de Chile: Diálogos del SITEAL, 2014.

SCHULTZ, T. Valor económico de la educación. México, D.F: Uthea, 1968.

SERRES, M. Pulgarcita. Paris: Le Pommier, 2012.

TAMAYO, A. El movimiento pedagógico en Colombia. Un encuentro de los maestros con la Pedagogía. Revista Histedbr, Campinas, n. 24, dez., p. 102-113, 2006.

Hacia una nueva visión del maestro. Revista Educación y Cultura. Bogotá, Fecode y Ceid, n. 10, diciembre, 1986.

TARDIF, M. El oficio docente en la actualidad. Perspectivas internacionales $\mathrm{y}$ desafíos de futuro. In: UNESCO. Seminario internacional: Políticas docentes. Formación, regulaciones y desarrollo profesional. Buenos Aires: UNESCO/IIEPI, 2012.

UNESCO. Replantear la educación: ¿Hacia un bien común mundial? París: Unesco, 2015.

. La educación encierra un tesoro. Informe a la Unesco de la Comisión Internacional sobre la educación para el siglo XXI. Paris: Santillana, 1996.

Declaração Mundial sobre Educação para Todos: satisfação das necessidades básicas de aprendizagem. Jomtien, 1990.

Recomendaciones 1934-1977. Conferencias Internacionales de Educación. Paris, 1966.

VEYNE, P. Foucault. Pensamiento y vida. Barcelona: Paidós Ibérica, 2009.

VIVES, J. Tratado de la enseñanza. Madrid: De la Lectura, 1923.

WEBER, M. Economía y sociedad. Madrid: FCE, 2002.

ZORA, L. F. V. Políticas docentes o de las tecnologías de formación, existencia y desaparición del maestro en Colombia. Tesis Doctoral. Belo Horizonte, Brasil: Universidade Federal de Minas Gerais. 2015

ZULUAGA, O. Las facultades de educación y el movimiento pedagógico. In: SUÁREZ, H. Veinte años del movimiento pedagógico. Bogotá: Cooperativa Editorial Magisterio, 2002.

. Pedagogía e historia: la historicidad de la pedagogía, la enseñanza, un objeto de saber. Bogotá: Siglo del Hombre, Anthropos, Universidad de Antioquia, 1999.

. Pedagogía e historia: la historicidad de la pedagogía, la enseñanza, un objeto de saber. Bogotá: Foro nacional por Colombia, 1987.

El maestro y el saber pedagógico en Colombia (1821-1848). Bogotá: Magisterio, 1984. 